\title{
ANÁLISE DA SECAGEM DE PARTÍCULAS DE ALUMINA EM SECADOR DE LEITO VIBROFLUIDIZADO
}

\author{
T. G. REIS ${ }^{1}$, H. PERAZZINI ${ }^{2}$, F. B. FREIRE ${ }^{1}$, J. T. FREIRE $^{1 *}$ \\ ${ }^{1}$ Universidade Federal de São Carlos, Departamento de Engenharia Química \\ ${ }^{2}$ Universidade Federal de Itajubá, Departamento de Engenharia Química \\ e-mail: freire@ufscar.br
}

\begin{abstract}
RESUMO
Foi realizado um estudo da secagem da alumina em leito vibrofluidizado. $O$ comportamento da secagem da alumina foi analisado em diferentes situações, variando a temperatura e a velocidade do ar de secagem, amplitude e frequência de vibração. No intervalo de condições experimentais utilizadas nos experimentos, observou-se: comportamentos distintos para cinética de secagem para um mesmo adimensional de vibração obtido através de duas combinações diferentes de amplitude e frequência; o modelo difusivo se ajustou bem aos dados experimentais, porém, sendo necessário antes analisar as condições de contorno utilizadas; os modelos semiempíricos que melhor se ajustaram aos dados experimentais foram o de Page (1974) e Overhults et al. (1973) e que uma única rede neural foi capaz de ajustar bem aos dados experimentais.
\end{abstract}

\section{INTRODUÇÃO}

O uso de ar seco em processos industriais tem se tornado cada vez mais frequente. Devido à variação meteorológica a umidade do ar ambiente está em constante variação, portanto é comum a utilização de colunas de adsorção para remoção da umidade contida no ar ambiente. Neste tipo equipamento é necessária a utilização de materiais, como a alumina, que são capazes de absorver esta umidade, sendo eles diferenciados por suas características como elevada área superficial e porosidade, o que o torna eficiente neste processo industrial. Após certo tempo em operação os materiais adsorventes ficam saturados, sendo então necessária sua recuperação para que possa ser reutilizado no processo de adsorção do ar, para tanto é necessário que se faça a secagem do material.

A secagem é um processo o qual deve ser criteriosamente analisado. Em materiais de elevada área superficial e porosidade, a secagem é controlada tanto por mecanismos convectivos quanto difusivos. Tal análise é de fundamental importância quando se deseja obter informações necessárias para o projeto, operação e controle do secador. Portanto este trabalho foi feito para contribuição do desenvolvimento da pesquisa investigando a secagem da alumina e associada a diferentes condições operacionais.

\section{MODELOS MATEMÁTICOS}

Existem na literatura diversos métodos para analisar e descrever a cinética de secagem de materiais, sendo os mais utilizados os modelos semiempíricos e o difusivo e o mais recentemente abordado a técnica de redes neurais, que apesar de não ter nenhum significado físico, tem apresentado excelentes resultados na representação da cinética de secagem. Estes modelos estão apresentados nos itens a seguir. 


\subsection{Equações semiempíricas}

As equações semiempíricas de modo geral baseiam-se na Lei de Newton do resfriamento aplicada à transferência de massa, o que presume que as condições sejam isotérmicas. As equações utilizadas neste trabalho foram: Lewis (1921), Brooker et al. (1974), Henderson \& Henderson (1968), Page (1949) e Overhults et al. (1973).

\subsection{Modelo difusivo}

De acordo com Brooker et al. (1992), nos métodos teóricos normalmente são consideradas as condições externas sob as quais a operação ocorre, assim como os mecanismos internos de transferência de calor e massa. Segundo Park et al. (2002) os modelos teóricos que descrevem a taxa de secagem decrescente consideram geralmente como mecanismo principal a difusão baseada em uma equação similar à lei de Fick, somente com o parâmetro difusividade efetiva no lugar da difusividade tradicional.

Para o modelo difusivo, ao fazer o balanço de massa e considerar a transferência de massa unidirecional (eixo z), obtêm-se a equação (1):

$$
\frac{\partial X(z, t)}{\partial t}=\frac{\partial}{\partial z}\left[D_{e f f} \frac{\partial X(z, t)}{\partial z}\right]
$$

Para obter a solução analítica do modelo difusivo, considera-se a difusividade efetiva de líquido e as propriedades do sólido constantes, conteúdo de umidade inicial uniforme e encolhimento do meio poroso desprezível. Desta forma é obtida a equação (2):

$$
\frac{\partial X(z, t)}{\partial t}=D_{\text {eff }, l} \frac{\partial^{2} X(z, t)}{\partial z^{2}}
$$

A equação (2) está sujeita às seguintes condições inicial e de contorno:

$$
\begin{array}{r}
\text { C.I.: } \begin{array}{r}
\left.X(\mathrm{z}, \mathrm{t})\right|_{\mathrm{x}=0} \\
=\mathrm{X}_{0}, \mathrm{t}=\mathrm{t}_{0}, 0<\mathrm{z} \\
<\mathrm{L}
\end{array} \\
\text { C.C.1: }\left.\left[\frac{\partial \mathrm{XX( \textrm {z } , \mathrm { t } )}}{\partial \mathrm{z}}\right]\right|_{\mathrm{z}=0}=0, \mathrm{t}>0
\end{array}
$$

A C.C.1 é geralmente aplicada em sistemas com geometria que se aproxima de uma placa plana de comprimento $2 \mathrm{~L}$ com $\mathrm{L}<\mathrm{z}<\mathrm{L}$ e que durante o processo de secagem, seca simetricamente tanto para $-\mathrm{L}<\mathrm{z}$ quanto para $\mathrm{z}<\mathrm{L}$. Em situações em que o meio poroso recebe uma corrente de ar ascendente na superfície inferior, deve ser considerada a hipótese de que não existe troca de massa da superfície inferior com o ambiente abaixo de $\mathrm{z}=0$.

Existem duas condições de contorno o qual podem ser consideradas em $\mathrm{z}=\mathrm{L}$. Para condições onde a resistência externa a transferência de massa é considerada desprezível, é considerado que se estabelece equilíbrio instantâneo na superfície superior do meio poroso $(\mathrm{z}=\mathrm{L})$, supondo a condição de contorno 2 (a).

$$
\begin{gathered}
\text { C.C.2(a): } \\
\left.X(z, t)\right|_{z=L}=X_{e q}, t>0
\end{gathered}
$$

A solução analítica para a equação (2) segundo as hipóteses e condições inicial e de contorno apresentadas, obtida por Crank (1975) através do método de separação de variáveis é:

$$
\begin{gathered}
X(t)=X_{\varepsilon q}+\left(X_{0}-X_{\varepsilon q}\right) \\
2 \sum_{n=0}^{\infty} \frac{(-1)^{n}}{\lambda_{n}} \cos \left(\ell \cdot \lambda_{n}\right) \exp \left(\lambda_{n}^{2} \frac{D_{\varepsilon f f . l}}{L^{2}} t\right)
\end{gathered}
$$

Uma vez que a concentração média espacial de umidade no meio poroso ao longo tempo é o que se obtém experimentalmente, o 
conteúdo de umidade média do meio poroso é obtido por:

$$
\bar{X}(\mathrm{t})=\frac{1}{L} \int_{0}^{L} X(z, t) d z
$$

Ao substituir a equação ((6) na equação (7) e realizar a integração, o conteúdo de umidade adimensional (YR) em função do tempo é:

$Y R=\frac{X_{t}-X_{\text {eq }}}{X_{0}-X_{\text {eq }}}=\frac{8}{\pi^{2}} \sum_{n=0}^{\infty} \frac{1}{(2 n+1)^{2}} \exp \left[-\left(n+\frac{1}{2}\right)^{2} \pi^{2} \frac{D_{\text {eff.l }}}{L^{2}} t\right]$

Quando a velocidade do gás altera a difusão efetiva de líquido, segundo Chen (2007), há outra condição de contorno que considera a convecção mássica na superfície do material, representada pela equação (9):

$$
\left.\left[\frac{\partial x(z, t)}{\partial z}\right]\right|_{z=L}=-\frac{\text { C.C.2(b): }}{h_{m, f_{g}, p \cdot \rho_{m}}}\left(Y_{\text {eq }}-Y_{\infty}\right), t>0
$$

De acordo com Cremasco (2008), ao considerar a condição de contorno de convecção e simetria é obtida a equação (10):

$$
\begin{aligned}
& Y R=\frac{X_{\mathrm{t}}-X_{\text {eq }}}{X_{0}-X_{\text {eq }}}= \\
& \sum_{n=1}^{\infty} \frac{2 \sin ^{2}\left(\lambda_{n}\right)}{\lambda_{n}^{2}+\lambda_{n} \sin \left(\lambda_{n}\right) \cos \left(\lambda_{n}\right)} \exp \left[-\lambda_{n}^{2} \frac{D_{\text {eff } .1}}{L^{2}} t\right]
\end{aligned}
$$

Os autovalores $\left(\lambda_{n}\right)$ podem ser conhecidos através da equação transcendental:

$$
B i_{m}=\lambda_{\mathrm{n}} \cdot \operatorname{tg}\left(\lambda_{\mathrm{n}}\right)
$$

\subsection{Redes Neurais}

Modelos de redes neurais artificiais têm sido usados com sucesso para predição de problemas na bioengenharia e na engenharia química (Baughman and Liu, 1995).
Segundo Movagharnejad et al (2007), as redes neurais artificiais são capazes de descrever um intervalo de experimentos enquanto as aplicações das correlações empíricas é limitada apenas para um experimento específico.

Redes neurais artificiais (RNAs) são sistemas que se assemelham às características de desempenho das redes neurais do cérebro humano. (Movagharnejad et al, 2007)

As redes neurais utilizam diversas células computacionais simples e interligadas, chamadas de neurônios. Os parâmetros computacionais são armazenados nos neurônios em unidades denominadas pesos sinápticos, e seus valores são modificados à medida que a rede sofre o processo de aprendizagem. Esta modificação ocorre para alcançar o valor alvo (Breve, 2006).

Patterson (1996), baseando-se na arquitetura das redes, definiu-as em três classes: redes aplicadas a camada única, redes aplicadas a multicamadas e redes neurais recorrentes.

\section{DETALHES EXPERIMENTAIS}

Neste procedimento as partículas também foram umidificadas por imersão em água por um período de três horas e então ficaram dispostas em um recipiente perfurado para eliminar o excesso de água.

Os procedimentos experimentais foram divididos em duas etapas, onde na primeira etapa o equipamento foi ligado e configurado para as condições operacionais desejadas, sendo elas de ${ }^{\Gamma}=4$ para combinações de amplitude e frequência de A: 0,003m e f: 18,2 $\mathrm{Hz}$ e A: $0,021 \mathrm{~m}$ e $6,8 \mathrm{~Hz}$. A altura do leito foi de $0,08 \mathrm{~m}$, temperatura do ar de $70,80 \mathrm{e}$ $100^{\circ} \mathrm{C}$ e velocidade do ar de secagem de 0,8Umvf, 1,0Umvf e 1,2Umvf. A condição de $\Gamma=4$ foi escolhida devido ao fato de que, no trabalho Meili (2009), foi o valor para o qual melhor se pôde observar comportamentos 
distintos do leito vibrofluidizado para um mesmo adimensional de vibração, obtido através de diferentes combinações de amplitude e frequência.

$\mathrm{Na}$ segunda parte, depois de atingidas condições de regime estacionário, as partículas foram inseridas na câmera de secagem até que as partículas atingissem uma altura de $7 \mathrm{~cm}$. Em intervalos de tempos prédeterminados, estipulado por um teste preliminar de cinética de secagem, foram retiradas do leito, com o auxílio de haste de ferro com recipiente na extremidade, amostras de aproximadamente $12 \mathrm{~g}$, para pesagem e então determinação da umidade do material feita pelo método direto da estufa, a $105^{\circ} \mathrm{C}$ $\pm 3^{\circ} \mathrm{C}$, por 24 horas. As pesagens foram feitas em uma balança analítica AND FR-200 MKII com precisão de $10-4 \mathrm{~g}$.

\section{ANÁliSE DOS RESULTADOS DE CINÉTICA DE SECAGEM}

A Figura 1 representa a cinética de secagem da alumina à temperatura de $80^{\circ} \mathrm{C}$ para $\Gamma=4$, porém comparada a duas configurações diferentes: a primeira para A: $0,003 \mathrm{~m}$ e f: $18,2 \mathrm{~Hz}$ (baixa amplitude) e a segunda para A: $0,021 \mathrm{~m}$ e $\mathrm{f}: 6,8 \mathrm{~Hz}$ (alta amplitude).

Figura 1: Cinética de secagem para leito vibro fluidizado a temperatura de $80^{\circ} \mathrm{C}$ e $\Gamma=\mathbf{4}$

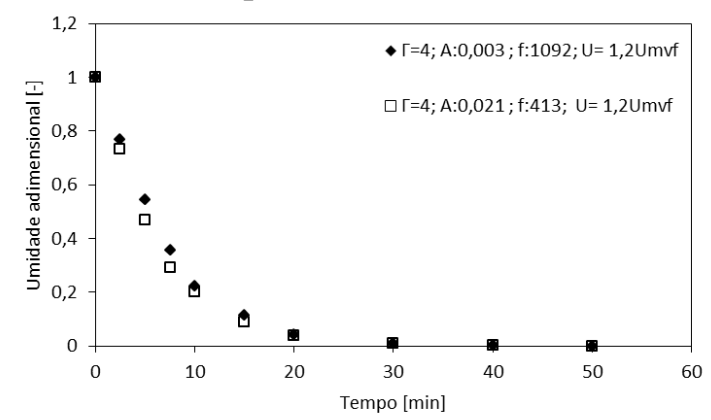

Através dos resultados experimentais, verifica-se que apesar da significativa diferença no comportamento fluidodinâmico, para a cinética de secagem, estas duas situações são bem próximas. Para a amplitude de A: $0,021 \mathrm{~m}$ a cinética é ligeiramente mais rápida. Entretanto, para este estado de vibração muitas das partículas foram danificadas.

Será apresentada a verificação do modelo difusivo utilizando a C.C.2(a) para temperatura e velocidade do ar de $70^{\circ} \mathrm{C}$ e 1,2 Umvf para configurações de vibração $\Gamma=4$ com A: $0,003 \mathrm{~m}$ e f: $18,2 \mathrm{~Hz}$, a qual está representada pela Figura 2.

Figura 2: Comparação entre os valores obtidos experimentalmente em leito vibrofluidizado para temperatura e velocidade do ar secagem de $70^{\circ} \mathrm{C}$ e Umvf, respectivamente, $\Gamma=4$; A: $0,003 \mathrm{~m} \mathrm{e}$ $\mathrm{f}: 18,2 \mathrm{~Hz}$ e previstos pelo modelo difusivo utilizando C.C.2(a)

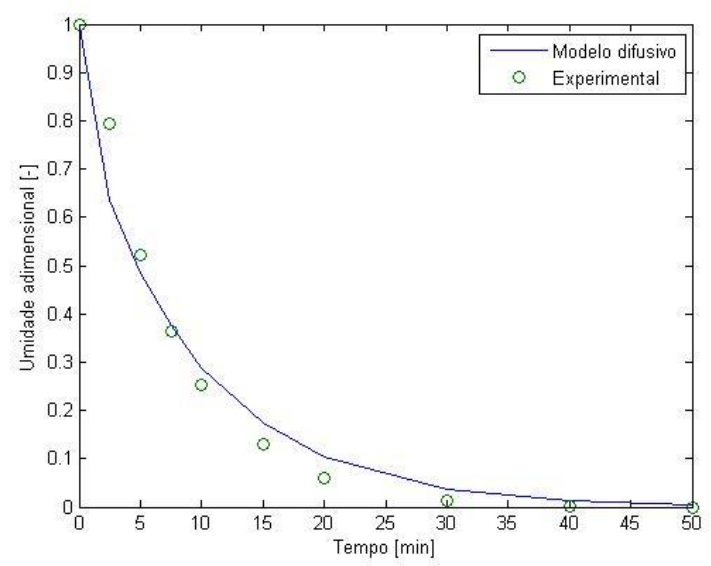

Conforme pode ser verificado pela Figura 2 o modelo apresentado não apresentou bom ajuste aos dados experimentais, visto que, no início do processo os dados de cinética de secagem estão subestimados e após aproximadamente seis minutos do início, os dados passam a ficar superestimados pelo modelo. A C.C.2(a) assume que a superfície do material está em equilíbrio termodinâmico com o meio, porém 
ao analisar a Figura 1 nota-se que a consideração feita ao modelo não apresenta bom ajuste aos dados experimentais. Desta forma, foi feito um novo ajuste aos dados experimentais utilizando a C.C.2(b), o qual considera a convecção mássica no processo, ajuste este o qual está representado pela Figura 3.

Figura 3: Comparação entre os valores obtidos experimentalmente em leito vibrofluidizado para temperatura e velocidade do ar secagem de $70^{\circ} \mathrm{C} \mathrm{e}$ 1,0Umvf, respectivamente, $\Gamma=4 ; \mathrm{A}: 0,003 \mathrm{~m} \mathrm{e}$ $\mathrm{f}: 18,2 \mathrm{~Hz}$ e previstos pelo modelo difusivo utilizando C.C.2(b)

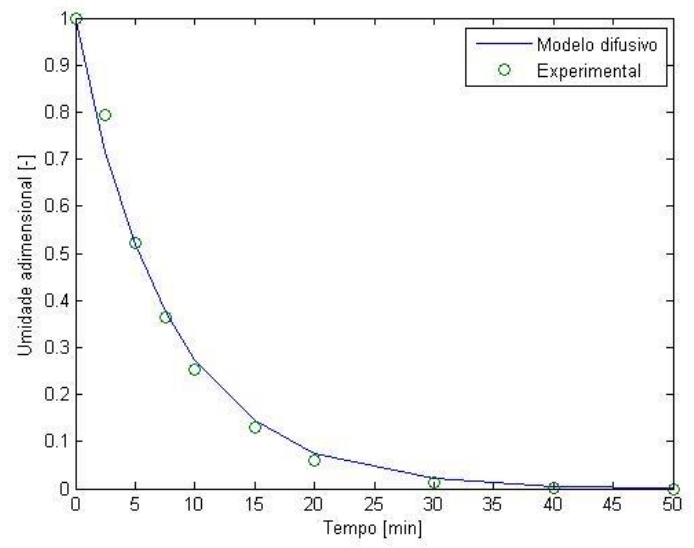

Nota-se na Figura 3 que a condição de contorno utilizada para fazer o ajuste do modelo aos dados experimentais, apresentou bom resultado, visto que este apresentou $\mathrm{r}^{2}=$ 0,9897, melhorando assim a representação da cinétia de secagem quando se compara a Figura 2.

A Figura 4 representa a comparação entre os valores obtidos experimentalmente através da secagem em leito vibrofluidizado para temperatura e velocidade do ar de secagem de $70^{\circ} \mathrm{C}$ e $1,2 \mathrm{Umvf}$ com $\Gamma=4$ sendo A: $0,003 \mathrm{~m}$ e f: $18,2 \mathrm{~Hz}$ e valores obtidos pelo modelo difusivo utilizando as C.C.2(a) e C.C.2.(b).
Figura 4: Comparação entre os valores obtidos experimentalmente em leito vibrofluidizado para temperatura e velocidade do ar secagem de $70^{\circ} \mathrm{C}$ e $1,2 \mathrm{U}_{\text {mvf }}$, respectivamente, $\Gamma=4 ; \mathrm{A}: 0,003 \mathrm{~m} \mathrm{e}$ $\mathrm{f}: 18,2 \mathrm{~Hz}$ e previstos pelo modelo difusivo utilizando C.C.2(a) e C.C.2(b)

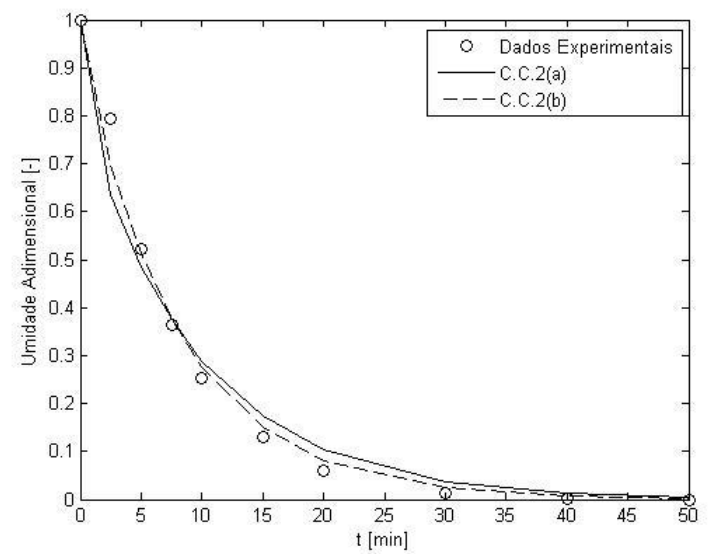

Conforme pode ser verificado pela Figura 4 os dados previstos pelo modelo utilizando a C.C.2(b) representa melhor os dados experimentais, pois as hipóteses consideradas estão mais próximas à realidade, se comparada a C.C.2(a). É possível dizer que o pequeno desvio ainda apresentado pelo modelo pode estar relacionado ao fato do modelo considerar a difusão efetiva como uma propriedade constante ao longo do processo, conforme já verificado por Perazzini (2014), e também relacionado ao fato de que as hipóteses consideradas pelo modelo não são realidades neste caso, como transferência de massa unidirecional, difusividade efetiva de líquido constante e camada fina, porém ainda assim o modelo é capaz de representar bem o processo de secagem.

Através do modelo difusivo também foi possível obter a difusividade efetiva para transferência de massa utilizando a equação (10). A solução foi obtida utilizando a ferramenta nlinfit do software MatLab. A partir dos dados apresentado na Tabela 1 nota-se que a temperatura do ar de secagem 
apresentou influência na Deff, para ambos os casos de combinações de amplitude e frequência. Verifica-se ainda que a velocidade do ar apresentou influência significativa para condição de baixa amplitude, porém não para situação de alta amplitude de vibração. Analisando preliminarmente a influência dos parâmetros vibracionais, nota-se que a difusividade efetiva depende de diferentes combinações de amplitude e frequência, ainda que se mantenha $\Gamma$ constante, o que indica a não universalidade do adimensional de vibração conforme já tinha sido observado por Perazzini (2013).
Tabela 1: Difusão efetiva prevista pelo modelo difusivo para secagem em leito vibrofluidizado

\begin{tabular}{ccccc}
\hline & \multicolumn{4}{c}{$\mathrm{D}_{\text {eff }}\left[\mathrm{m}^{2} / \mathrm{s}\right]$} \\
\cline { 2 - 5 } $\mathrm{T}\left[{ }^{\circ} \mathrm{C}\right]$ & \multicolumn{2}{c}{$1,0 \mathrm{U}_{\text {mvt }}$} & \multicolumn{2}{c}{$1,2 \mathrm{U}_{\text {mvt }}$} \\
& $\mathrm{A}:$ & $\mathrm{A}:$ & $\mathrm{A}:$ & $\mathrm{A}:$ \\
& $0,003 \mathrm{~m}$ & $0,021 \mathrm{~m}$ & $0,003 \mathrm{~m}$ & $0,021 \mathrm{~m}$ \\
\hline & $4,54 \mathrm{E}-$ & $5,57 \mathrm{E}-$ & $5,50 \mathrm{E}-$ & $5,63 \mathrm{E}-$ \\
70 & 04 & 04 & 04 & 04 \\
& $5,04 \mathrm{E}-$ & $6,32 \mathrm{E}-$ & $5,69 \mathrm{E}-$ & $6,54 \mathrm{E}-$ \\
80 & 04 & 04 & 04 & 04 \\
& $5,96 \mathrm{E}-$ & $7,89 \mathrm{E}-$ & $6,88 \mathrm{E}-$ & $8,53 \mathrm{E}-$ \\
100 & 04 & 04 & 04 & 04 \\
\hline
\end{tabular}

Após a utilização do modelo difusivo, os dados foram também ajustados aos modelos semiempíricos apresentados no item 2.1, onde foi utilizado o método da soma dos mínimos quadrados para ajuste das curvas. Por apresentarem valor de $\mathrm{r}^{2}$ mais próximos de 1 e menores SQR e EQM nota-se que as curvas que melhor se ajustaram aos pontos experimentais em todas as condições experimentais foram as originadas dos modelos de Page (1945) e Overhults et al (1975).

Tabela 2: Resultados estatísticos para ajuste dos modelos empíricos aos dados experimentais para cinética de secagem em leito vibrofluidizado

\begin{tabular}{ccccccc}
$\begin{array}{c}\text { Temperatura } \\
{\left[{ }^{\circ} \mathbf{C}\right]}\end{array}$ & $\mathbf{A}[\mathrm{m}]$ & Velocidade & \multicolumn{1}{c}{ Modelo } & $\mathbf{r}^{2}$ & SQR & EQM \\
\hline & & & Lewis & 0,9968 & 0,0052 & $5,23 \mathrm{E}-04$ \\
& & & Brooker et al. & 0,9964 & 0,0047 & $4,71 \mathrm{E}-04$ \\
70 & 0,003 & \multirow{2}{*}{$\begin{array}{l}\text { Henderson } \& \\
\text { mvf }\end{array}$} & $\begin{array}{l}\text { Henderson } \\
\text { Henderson }\end{array}$ & 0,9964 & 0,0047 & $4,71 \mathrm{E}-04$ \\
& & & Page & 0,9986 & 0,0016 & $1,61 \mathrm{E}-04$ \\
& & & Overhults et al. & 0,9986 & 0,0016 & $1,61 \mathrm{E}-04$ \\
\hline
\end{tabular}

Além da utilização do modelo difusivo e dos modelos empíricos, também foi utilizada a ferramenta de ajuste de redes neurais, a qual foi criada utilizando o comando nftool do software MathworksMatlab, da mesma maneira utilizada para secagem em camada fina. Para cinética de secagem em leito vibrofluidizado foi criada uma única rede neural para todo o conjunto de dados experimentais com uma camada de entrada de cinco neurônios onde as variáveis fornecidas foram o tempo de processo, temperatura e velocidade do ar de secagem, amplitude e frequência de vibração. A rede possui também uma camada intermediária com cinco neurônios e uma 
camada de saída com um neurônio cuja variável fornecida é a umidade. O treinamento da rede foi feito com cem épocas utilizando $60 \%$ das informações do banco de dados experimental e a verificação e o teste da rede foram feitos utilizando $20 \%$ dos dados cada um respectivamente.

A Figura 5 e representa os dados experimentais e o ajuste proposto pelo modelo das redes neurais para temperatura e velocidade do ar de secagem de $70^{\circ} \mathrm{C}$ e 1,0 Umvf, para $\Gamma=4$ e A: $0,003 \mathrm{~m}$ e f: $18,2 \mathrm{~Hz}$ e $100^{\circ} \mathrm{C}$.

Figura 5: Comparação entre os valores obtidos experimentalmente e previstos pelo modelo de redes neurais - para secagem em leito vibrofluidizado a temperatura e velocidade de $70^{\circ} \mathrm{C}$ e $1,0 \mathrm{U}_{\mathrm{mvf}} \Gamma=4$ e A:0,003m e f: $18,2 \mathrm{~Hz}$

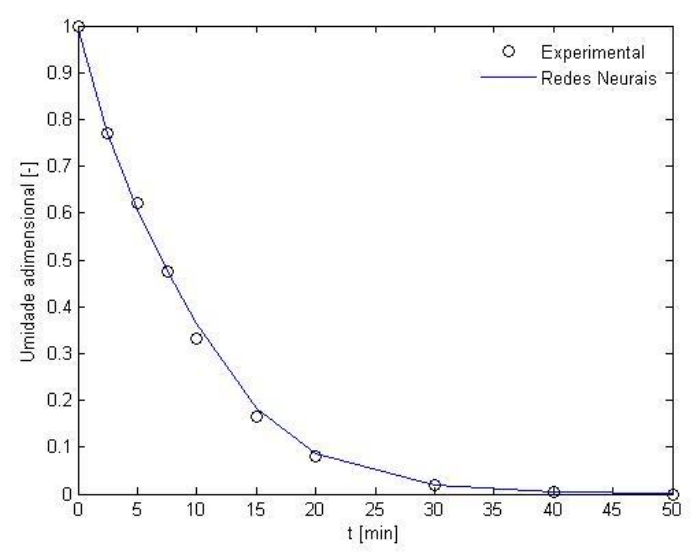

Como é possível visualizar através da Figura 5, o uso da técnica de redes neurais mostrou-se muito eficiente, visto que uma única rede foi capaz de reproduzir bem os dados de cinética de secagem em função do tempo.

Além do bom ajuste aos dados experimentais, as redes neurais mostraram-se eficientes também para simular resultados experimentais dentro da faixa de operação na qual os experimentos foram realizados. A Figura 6 representa os dados experimentais da cinética de secagem em função do tempo para temperatura do ar de secagem de $70^{\circ} \mathrm{C}$, velocidade de 1,0Umvf e 1,2Umvf e também a simulação feita para $1,1 \mathrm{Umvf}$ para $\Gamma=4 \mathrm{e}$ A: $0,003 \mathrm{~m}$ e f: $18,2 \mathrm{~Hz}$. Esses dados foram obtidos fora dos valores usados para treinamento e verificação da rede, porém no intervalo de valores usados neste trabalho.

Figura 6: Comparação entre os valores obtidos experimentalmente para velocidade do ar de secagem de $1,0 \mathrm{U}_{\mathrm{mvf}}$ e $1,2 \mathrm{U}_{\mathrm{mvf}}$ respectivamente e temperatura de $70^{\circ} \mathrm{C}$ e dados simulados pelo modelo de redes neurais para $1,1 \mathrm{U}_{\text {mvf }}$ em leito vibrofluidizado

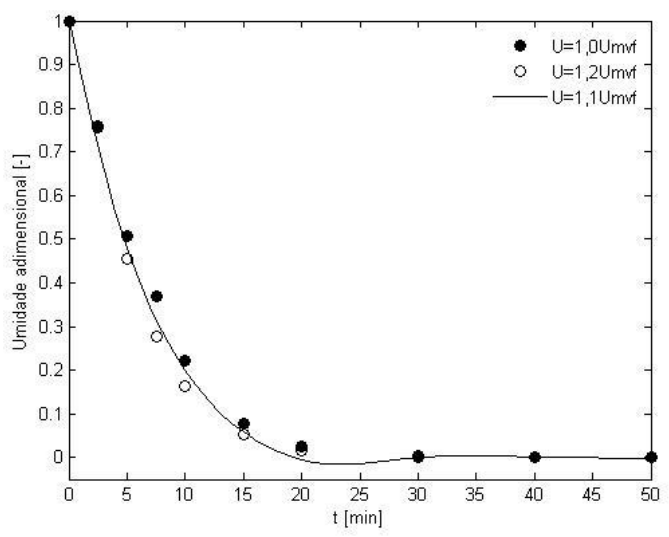

Como forma de comparação entre os modelos testados para descrever a cinética de secagem em leito vibrofluidizado, estão apresentadas na Figura 7 as cinéticas de secagem em função do tempo obtidas através dos dados experimentais, modelo difusivo, semiempíricos e das redes neurais. A Figura 7 mostra os dados para temperatura e velocidade do ar de secagem de $70^{\circ} \mathrm{C}$ e $1,2 \mathrm{Umvf}, \mathrm{com}^{\Gamma}=4$ e A: $0,003 \mathrm{~m}$ e f: $18,2 \mathrm{~Hz}$. 
Figura 7: Comparação entre os valores obtidos experimentalmente e previsto pelos modelos difusivo C.C.2(b), Overhults et al. (1973) e redes neurais para secagem em leito vibrofluidizado para temperatura de $70^{\circ} \mathrm{C}$ e $1,2 \mathrm{Umvf} \mathrm{com}=4$ e A:0,003m e f: $18,2 \mathrm{~Hz}$

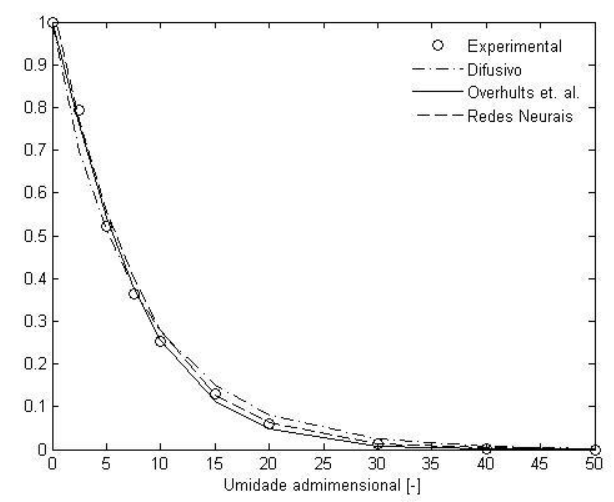

\section{NOMENCLATURA}

$\begin{array}{lll}\text { A } & \text { Amplitude de vibração } & {[\mathrm{L}]} \\ \mathrm{D}_{\text {eff }} & \text { Difusividade efetiva } & {\left[\mathrm{L}^{2} \mathrm{~T}^{-1}\right]} \\ \mathrm{f} & \text { Frequência de vibracão } & {\left[\mathrm{T}^{-1}\right]} \\ \mathrm{L} & \text { Espessura } & {[\mathrm{L}]} \\ \mathrm{t} & \text { Tempo } & {[\mathrm{T}]} \\ \mathrm{U} & \text { Velocidade do ar } & {\left[\mathrm{LT}^{-1}\right]} \\ & \text { Velocidade de mínima } & \\ \mathrm{U}_{\mathrm{mf}} & \text { fluidização } & {\left[\mathrm{LT}^{-1}\right]} \\ & \text { Velocidade de mínima } & \\ \mathrm{U}_{\mathrm{mvf}} & \text { vibrofluidização } & {\left[\mathrm{LT}^{-1}\right]} \\ \mathrm{X} & \text { Umidade em base seca } & {\left[\mathrm{MM}^{-1}\right]} \\ \overline{\bar{X}} & \text { Umidade média } & {\left[\mathrm{MM}^{-1}\right]} \\ \mathrm{X}_{0} & \text { Umidade inicial } & {\left[\mathrm{MM}^{-1}\right]} \\ \mathrm{X}_{\mathrm{cr}} & \text { Umidade crítica } & {\left[\mathrm{M} \mathrm{M}^{-1}\right]} \\ \mathrm{X}_{\mathrm{eq}} & \text { Umidade de equilíbrio } & {\left[\mathrm{M} \mathrm{M}^{-1}\right]} \\ Y_{\infty} & \text { Umidade do gás de } & {\left[\mathrm{M} \mathrm{M}^{-1}\right]} \\ & \text { secagem } & \end{array}$

YR Umidade adimensional [-]

z Coordenada espacial [L]

$\Gamma \quad$ Adimensional de vibração [-]

$\lambda_{n} \quad$ Raízes da equação

transcendental

$\omega \quad$ Frequência angular $\quad\left[\mathrm{T}^{-1}\right]$

Comprimento

característico

\section{REFERÊNCIAS}

BAUGHMAN, D.R., LIU, Y. A., 1995. Neural Networks in Bio-Processing and Chemical Engineering. Academic Press. New York.

BROOKER, D. B., BAKKER-ARKEMA, F. W., HALL, C. W., Drying cereal grains, The Avi Publishing Company, Inc., Westport, 1974.

BROOKER, D. B., BAKKER-ARKEMA, F. W., HALL, C. W., Drying and storage of grains and oilseeds. New York. AVI Book, 1992. 450p.

CHEN, X. D. Moisture diffusivity in food and biological materials. Drying Technology, v.25, p.1203-1213, 2007.

CRANK, J. The mathematics of diffusion. 2. Ed. Oxford: Claredon Press, 1975. 414p.

CREMASCO, M. A. Fundamentos de transferência de massa. 2. Ed. Campinas: Editora Unicamp, 2008. 725 p.

HENDERSON, J. M, HENDERSON, S. M. A computational procedure for deep 
bed drying analysis, J. Agric. Eng. Res., Vol. 13, p.87-95, 1968.

LEWIS, W. K. The rate of drying of solids material. In: The Symposium on Drying. The Journal of I. \& E. Ch. Vol. 13 (5), p. 427-432, 1921.

MOVAGHARNEJAD, K; NIKZAD,M. Modeling of tomato drying using artificial neural network. Computers and Eletronics in Agriculture, v. 59, p. 7885. 2007.

OVERHULTZ, D. G., WHITE, G. M., HAMILTON, H. E., ROSS, I. J., Drying of soybeans with heated air, Transactions of ASAE, Vol. 16, p. 112-113, 1973.

PAGE, C., Factors influencing the maximum rate of drying shelled corn in layers, $M$. S. Thesis, Purdue University, 1949.

PARK, K. J., VOHNIKOZA, Z.; BROD, F. $P$. R. Evaluation of drying parameters and desorption isotherms of garden mint leaves (Menthacrispa L.) Journal of Food Engineering. Davis, v.51, n.3, p.193-199, 2002.

PERAZZINI, H., FREIRE, F.B., FREIRE, J. T., Difusão de umidade na secagem de partículas porosas em leito vibrofluidizado. Anais do XXXVI Congresso Brasileiro de Materiais Particulados. Maceió: Universidade Federal de Alagoas, 2013.

PERAZZINI, H., Secagem de sólidos granulares. 2014. 170p. (Tese de Doutorado em Engenharia Química) Departamento de Engenharia Química, Universidade Federal de São Carlos, SP, 2014. 